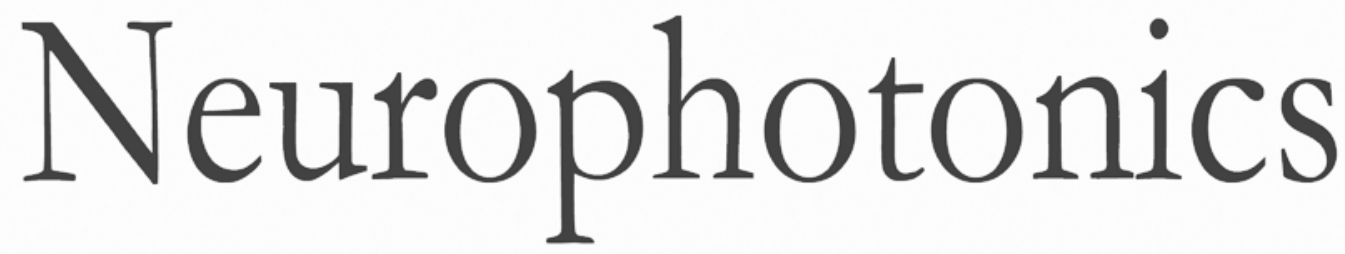

\title{
Super-resolution imaging and estimation of protein copy numbers at single synapses with DNA-point accumulation for imaging in nanoscale topography
}

Carolin Böger

Anne-Sophie Hafner

Thomas Schlichthärle

Maximilian T. Strauss

Sebastian Malkusch

Ulrike Endesfelder

Ralf Jungmann

Erin M. Schuman

Mike Heilemann 


\title{
Super-resolution imaging and estimation of protein copy numbers at single synapses with DNA-point accumulation for imaging in nanoscale topography
}

\author{
Carolin Böger, ${ }^{a}$ Anne-Sophie Hafner, ${ }^{b}$ Thomas Schlichthärle, ${ }^{c, d}$ Maximilian T. Strauss, ${ }^{c, d}$ Sebastian Malkusch, ${ }^{a}$ \\ Ulrike Endesfelder, ${ }^{e}$ Ralf Jungmann, ${ }^{c, d}$ Erin M. Schuman, ${ }^{b, *}$ and Mike Heilemann ${ }^{\mathrm{a}, *}$ \\ ${ }^{a}$ Goethe University, Institute of Physical and Theoretical Chemistry, Frankfurt, Germany \\ bMax Planck Institute for Brain Research, Frankfurt, Germany \\ 'Ludwig Maximilian University, Center for Nanoscience, Faculty of Physics, Munich, Germany \\ ${ }^{d}$ Max Planck Institute of Biochemistry, Martinsried, Germany \\ eMax Planck Institute for Terrestrial Microbiology, Marburg, Germany
}

\begin{abstract}
In the brain, the strength of each individual synapse is defined by the complement of proteins present or the "local proteome." Activity-dependent changes in synaptic strength are the result of changes in this local proteome and posttranslational protein modifications. Although most synaptic proteins have been identified, we still know little about protein copy numbers in individual synapses and variations between synapses. We use DNA-point accumulation for imaging in nanoscale topography as a single-molecule super-resolution imaging technique to visualize and quantify protein copy numbers in single synapses. The imaging technique provides near-molecular spatial resolution, is unaffected by photobleaching, enables imaging of large field of views, and provides quantitative molecular information. We demonstrate these benefits by accessing copy numbers of surface AMPA-type receptors at single synapses of rat hippocampal neurons along dendritic segments. $\odot$ The Authors. Published by SPIE under a Creative Commons Attribution 4.0 Unported License. Distribution or reproduction of this work in whole or in part requires full attribution of the original publication, including its DOI. [DOI: 10.1117/1.NPh.6.3.035008]
\end{abstract}

Keywords: synaptic proteins; super-resolution imaging; molecular quantification; DNA-point accumulation for imaging in nanoscale topography.

Paper 19031LRR received Mar. 29, 2019; accepted for publication Jul. 31, 2019; published online Aug. $21,2019$.

\section{Introduction}

Neurons are highly specialized cells that communicate with one another at synapses, points of close contact between the axon of one neuron and the dendrite of another. The 1 to 10,000 synapses within a typical single dendritic arbor can greatly vary in shape and size, reflecting differences in their synaptic efficacies. ${ }^{1}$ A single synapse likely contains hundreds of different protein types. Changes in the copy number, distribution, and/or turnover of these proteins within an individual synaptic compartment will directly affect synaptic transmission. These same variables are tuned to bring about the changes in synaptic strength associated with synaptic plasticity.

The heterogeneity of protein distribution at different synapses, positioned from 10 to $800 \mu \mathrm{m}$ from the neuronal cell body, is largely unexplored. Although good estimates of the composition of an average synapse exist, ${ }^{2}$ little is known about the heterogeneity of protein organization and relative copy number between proteins or between synapses. It is known that the integrative properties of dendrites and synapses change as a function of distance from the neuronal cell body, but the proteomic landscape of synapses within a single cell is not known. This gap is largely due to limitations in existing methods for quantifying and mapping proteins at the whole-cell level. Indeed, multiple methods for quantifying proteins in single cells have been developed, including single-cell Western blots, ${ }^{3} \mathrm{CyTOF}^{4}$ and Proseek Multiplex. ${ }^{5}$ However, when these methods enable the

*Address all correspondence to Erin M. Schuman, E-mail: erin.schuman @ brain .mpg.de; Mike Heilemann, E-mail: heilemann@chemie.uni-frankfurt.de quantification of endogenous proteins, they lack the ability to localize those proteins in intact cells.

Recent advances in optical microscopy have opened the door to imaging cell biology with molecular resolution. ${ }^{6}$ Among the various techniques available, single-molecule localization microscopy (SMLM) is unique in that it achieves subdiffraction spatial localization and at the same time provides quantitative information. ${ }^{7}$ Molecular counting with SMLM was originally demonstrated using stationary labels with photoswitchable fluorophores. ${ }^{8}$ This approach has two major limitations: first, it suffers from photobleaching within and around the field of view (Fig. S1 in Supplementary Material), which prevents imaging of large field of views. Second, low numbers of singlemolecule events can affect the accuracy of molecular quantification, ${ }^{9,10}$ and the resulting inaccuracy is exacerbated by the typical isolated fields of view. ${ }^{11}$ Both limitations can be bypassed by DNA-point accumulation for imaging in nanoscale topography (DNA-PAINT), ${ }^{12}$ an extension of the original concept of PAINT $^{13}$ that built on the repetitive and transient binding of a fluorophore to a target. In DNA-PAINT, short (9 to $10 \mathrm{nt}$ ) fluorophore-labeled oligonucleotides ("imager strands") transiently bind to a target oligonucleotide ("docking strand") conjugated to a labeling probe, e.g., an antibody, affimer, or aptamer, ${ }^{12}$ targeting a protein-of-interest. Transient binding of the imager strand to the docking strand generates a stationary fluorescence signal, which enables the localization of single fluorophores and the generation of a super-resolved image. The association kinetics of the DNA duplex formation is known and relatively well-defined in many experimental settings, allowing for a straightforward quantification of the number of molecular 
(a)

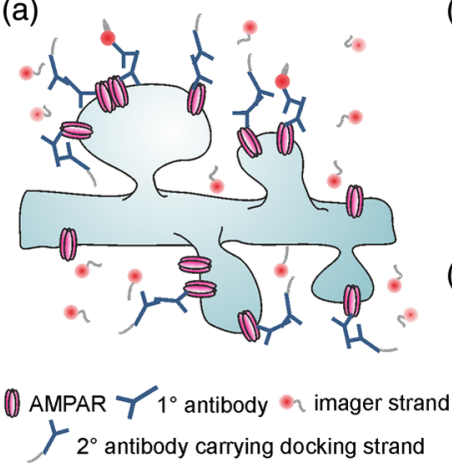

(b)

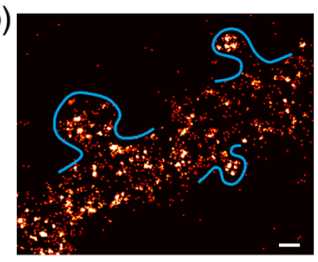

(c)

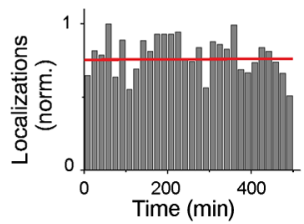

(d)
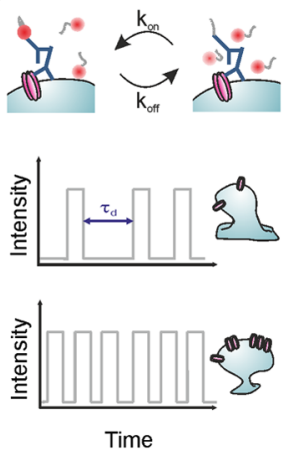

Fig. 1 DNA-PAINT imaging of synaptic proteins: (a) scheme of protein labeling for DNA-PAINT using antibodies. A secondary antibody is decorated with a docking strand, in which a fluorophore-labeled complementary imager strand binds transiently and generates the single-molecule signal, (b) superresolved DNA-PAINT image of GluA2-containing AMPAR with single synapses assigned manually (scale bar $500 \mathrm{~nm}$ ), (c) the number of single-molecule localizations in a DNA-PAINT experiment is constant over long acquisition times, and (d) local protein copy numbers are determined from the association rate of the imager strand binding to the target strand $\left(k_{\mathrm{on}}\right)$, which is the inverse of the time between binding events (dark time, $\tau_{d}$ ). The number of antibody-labeled proteins in a synapse is related to $1 / \tau_{d}$.

targets using a variation of DNA-PAINT called quantitative PAINT or qPAINT. ${ }^{14}$ A nanomolar concentration of the imager strand in the imaging buffer guarantees a constant exchange of labels, which makes DNA-PAINT insensitive to photobleaching and allows imaging of large field of views. In theory, datasets of infinite length can be recorded allowing for robust molecular quantification. $^{14}$

Here, we introduce DNA-PAINT imaging of synaptic proteins in neurons. We demonstrate bleaching-insensitive imaging of large fields of view, together with robust molecular quantification. As a proof of concept, we determine copy numbers of GluA2, an integral component of the (AMPA-type) glutamate receptor complex (AMPAR), in single synapses and across dendrites.

\section{Results}

Here we establish super-resolution imaging and quantification of synaptic proteins with DNA-PAINT ${ }^{12}$ [Fig. 1(a)]. DNAPAINT uses the repetitive and transient binding of a fluorophore-labeled imager strand to a target-bound docking strand. We used DNA-PAINT to map and quantify the distribution of one AMPAR subunit, GluA2, on the surface of neuronal dendrites [Fig. 1(b)]. We found a constant number of single-molecule localizations over time [Fig. 1(c)], which demonstrates that this approach is bleaching-insensitive and suitable for imaging large field of views. An additional benefit of DNA-PAINT is molecular quantification, which is accessible from the analysis of the DNA binding kinetics and termed qPAINT. ${ }^{14}$ In brief, the time interval between binding events (dark time $\tau_{d}$ ) inversely scales with the number of labeled targets in that area [Fig. 1(d)]. Calibrating the dark time of DNA-PAINT-labeled protein clusters in synapses with a known single-binding site allows molecular quantification.

In order to explore the range of protein densities that are accessible using this method, we generated simulated qPAINT data mimicking densely packed protein clusters. We simulated qPAINT data of DNA origami with 20 targets spaced either 40 [Fig. 2(a)] or $15 \mathrm{~nm}$ apart [Fig. 2(b)] (for simulation parameters, see Sec. 3), which we call synthetic protein clusters. Grouping of three of these synthetic protein clusters mimics the previously reported nano-organization of AMPAR in single synapses ${ }^{15}$
[Figs. 2(a) and 2(b)]. In order to extract protein copy numbers from these synthetic clusters, we followed the published protocol of qPAINT analysis. ${ }^{12,14}$ We first determined the average dark time of a single binding site in the simulated synthetic clusters $\tau_{d \text {,single }}$ as $1266 \mathrm{~s}$. We next determined the average dark time of whole synthetic clusters $\tau_{d \text {,cluster }}$. We calculated the number of detected targets within a synthetic cluster by calculating the ratio of $\tau_{d \text {,single }} / \tau_{d \text {,cluster }}$ (see Sec. 3) [Figs. 2(a) and 2(b)]. For both 15and $40-\mathrm{nm}$ spaced targets in the synthetic clusters, qPAINT analysis of the simulated data reports the same number of $\sim 16.8$ detected targets [Figs. 2(a) and 2(b), numbers next to synthetic clusters]. Compared to a ground truth of 20 targets in each synthetic cluster, we underestimate the number of targets by $\sim 16 \%$. This underestimation results from the comparatively low imager strand concentration of $500 \mathrm{pM}$ in combination with single-site calibration employed here. Absolute numbers of DNAlabeled secondary antibodies are determined straight forward by correcting with this factor. We note that this underestimation is not an intrinsic qPAINT bias but rather a consequence of the experimental calibration strategy and parameters used for
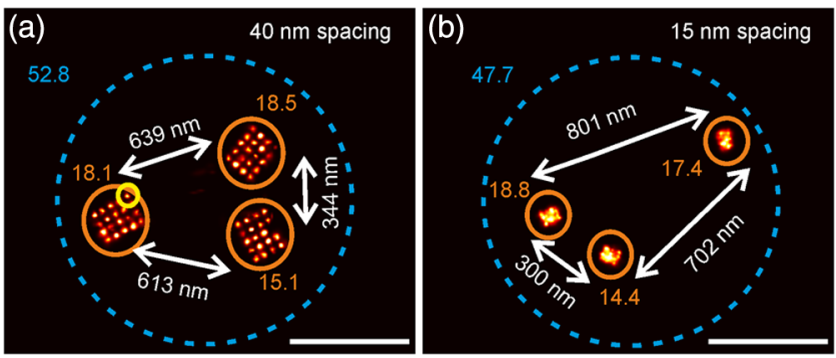

Fig. 2 Simulation of DNA-PAINT data and qPAINT analysis. (a), (b) DNA origami patterns containing 20 target sites spaced (a) $40 \mathrm{~nm}$ and (b) $15 \mathrm{~nm}$ apart were simulated as synthetic clusters of synaptic proteins. A dark time analysis of single docking strands [yellow circle in (a)] yielded $\tau_{d \text {,single }}$. Copy numbers of single synthetic clusters (orange circles and numbers) and of assemblies of three clusters (blue circles and numbers) were determined by extracting $\tau_{d, \text { cluster }}$ and calibrating with $\tau_{d \text {,single }}$. Using simulation parameters that match the expected range of AMPAR clustering, a detection efficiency of 0.84 was determined (scale bar $500 \mathrm{~nm}$ ). 
mimicking our in situ experiments. To illustrate that this underestimation indeed stems from our calibration strategy, we compared simulated data employing single-site calibration as well as single-structure calibration (i.e., 20 sites), showing that in the latter case, we can indeed recover the correct number of binding sites per structure (see Fig. S2 and Supplementary Note 1 in Supplementary Material). We also analyzed groups of three synthetic clusters and extracted copy numbers [Figs. 2(a) and 2(b), numbers next to large circles].

We next live-labeled GluA2 in cultured rat hippocampal neurons using an antibody against the N-terminal (surface

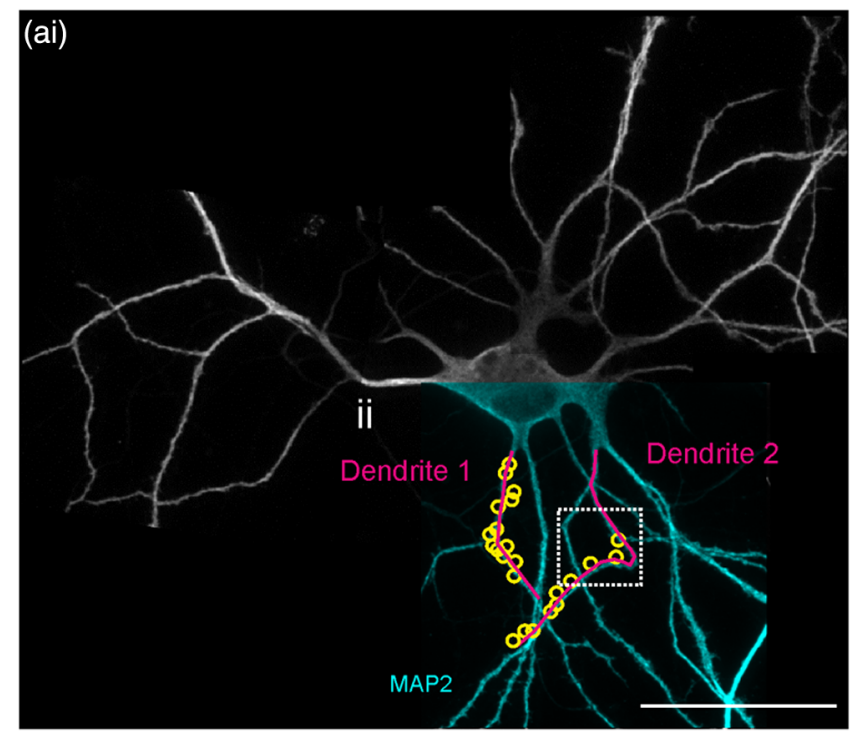

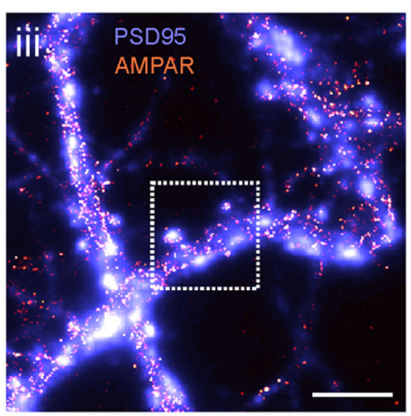

(b)

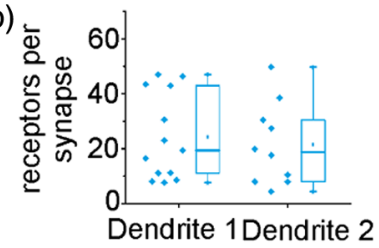

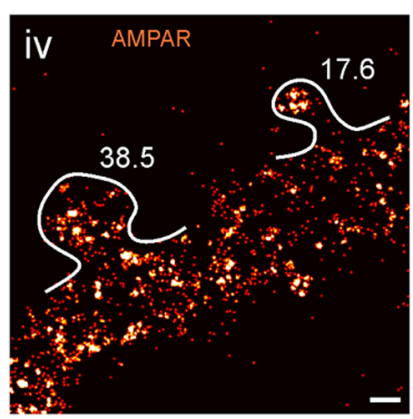

(c)

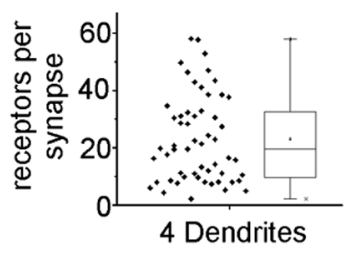

Fig. 3 Quantitative PAINT imaging of GluA2-containing AMPAR in single synapses: (a) large images (i) are generated by tiling multiple single confocal images, (ii) using immunostained MAP2 as a dendritic marker. Insets show (iii) co-staining of synapses with PSD95, and (iv) a zoom-in of the DNA-PAINT image of GluA2-containing AMPARs with numbers indicating detected AMPARs; (b) copy numbers of GluA2-containing AMPAR in single synapses along two single dendrites highlighted in (ii) [yellow circles in (ii) mark the location of single synapses analyzed], yielding 24 ( \pm 16 s.d., $n=13$ synapses; dendrite 1) and 21 ( \pm 15 s.d., $n=10$ synapses; dendrite 2 ); (c) the analysis of 56 synapses in 4 dendrites from two neurons ( 2 dendrites per neuron) yielded an average number of 23 ( \pm 15 s.d.) GluA2-containing AMPA receptors per synapse (scale bars $80 \mu \mathrm{m}$ and $500 \mathrm{~nm}$ ). exposed) part of the GluA2, fixed the sample, and then added a secondary antibody coupled to a DNA docking strand (see Sec. 3). In order to cover large fields of view, we also immunolabeled neurons for MAP2 as dendritic marker and obtained confocal images of neighboring regions which we tiled together [Figs. 3(ai) and 3(aii)]. In these large images, we identified single dendrites that next were imaged with DNA-PAINT. We visualized synaptic and extra-synaptic GluA2 with subdiffraction resolution [Fig. 3(aiii)] and determined an experimental localization precision of $17.4 \mathrm{~nm} .{ }^{16}$ We observed clusters of GluA2 within dendritic spines and on the dendritic shaft. In order to eliminate signal occurring from nonspecific binding of the imager strands, we filtered single-molecule localizations by the known binding kinetics of the DNA duplex. This important step rules out the detection of artificial clusters of proteins, which with other SMLM methods would not be possible and go into the quantitative analysis (Supplemental Note 1 and Fig. S3 in Supplementary Material). We next analyzed the number of GluA2 molecules in single synapses and along dendrites by qPAINT. For this purpose, we selected small and isolated spots along the dendritic shaft as calibration foci that likely represent single AMPA receptors [Fig. S3(d) in Supplementary Material], which were visualized using the same oligo-labeled antibody and imager strand. In order to identify synapses, we used PSD95, an integral protein of postsynaptic densities, ${ }^{17}$ and overlaid confocal and DNA-PAINT images (see Sec. 3). For the two dendrites shown in Fig. 3(a), we found 24 ( \pm 16 s.d., $n=13$ synapses) and 21 ( \pm 15 s.d., $n=10$ synapses) GluA2-containing AMPA receptors per synapse, respectively [Fig. 3(b)]. The analysis of 56 synapses in 4 dendrites from 2 neurons yielded an average number of 23 ( \pm 15 s.d.) GluA2-containing AMPA receptors per synapse. These results are in agreement with previously reported quantitative super-resolution data, using the same primary antibody. ${ }^{15}$ Following this previous study, we note that we developed quantitative imaging of proteins in neurons for secondary antibodies, and hence provide a tool for absolute quantification of DNA-labeled antibodies in neurons. For a direct quantification of target proteins, we recommend the use of DNA-labeled primary antibodies with a known number of DNA strands per antibody.

At excitatory synapses, the number and position of glutamate receptors within the synapse is perhaps the most important determinant of synaptic function and variation in AMPAR number is thought to underlie numerous forms of synaptic plasticity; in particular, long-term potentiation in the hippocampus. Superresolution approaches have been used to assess AMPAR nanoorganization and copy number within synapses..$^{15}$ Here we used DNA-PAINT $^{12}$ to map and quantify the distribution of one AMPAR subunit, GluA2, on the surface of neuronal dendrites. We present a robust protocol for quantitative super-resolution imaging in entire individual neurons using DNA-PAINT. This approach has several key advantages that were particularly tailored for the application in quantitative super-resolution imaging neurons: first, it is insensitive to photobleaching and allows imaging of large sample sizes. Second, we use a kinetic filter to discriminate true signal from background signal, which minimizes false localizations and is only possible with DNAPAINT. Third, we extract absolute numbers of fluorophorelabeled antibodies, which is the prerequisite for absolute quantification using stoichiometric protein labels. Future work might further expand this technology to multiplexed, quantitative super-resolution imaging of whole neurons. 


\section{Materials and Methods}

\subsection{Hippocampal Neurons}

Dissociated rat hippocampal neuron cultures were prepared and maintained essentially as described previously. ${ }^{18,19}$ Briefly, we dissected hippocampi from postnatal day 0 to 1 rat pups of either sex (Sprague-Dawley strain; Charles River Laboratories), dissociated them with papain (Sigma) and plated them at a density of $30 \times 10^{3}$ cells $/ \mathrm{cm}^{2}$ on poly-D-lysine-coated glass-bottom Petri dishes (MatTek). Hippocampal neurons were maintained and matured in a humidified atmosphere at $37^{\circ} \mathrm{C}$ and $5 \% \mathrm{CO}_{2}$ in growth medium (Neurobasal-A supplemented with B27 and GlutaMAX-I, Life Technologies) for 18 to 21 days in vitro (DIV) to ensure synapse maturation. All experiments complied with national animal care guidelines and the guidelines issued by the Max Planck Society and were approved by local authorities.

\subsection{Immunolabeling of Cultured Neurons}

Glass bottom dishes with attached neurons (DIV 18 to 21) were incubated with a mouse monoclonal antibody anti-GluA2 specifically binding to a $\mathrm{N}$-terminal extracellular epitope of the subunit for $8 \mathrm{~min}$ at $37^{\circ} \mathrm{C}$ and $5 \% \mathrm{CO}_{2}$ in original growth medium $(0.24 \mu \mathrm{g} / \mathrm{ml}$, gift from E. Gouaux, Vollum Institute, Portland). Neurons were washed twice in warm original growth medium and once in room temperature PBS containing $2 \mathrm{mg} \mathrm{CaCl}$ and $2 \mathrm{mg} \mathrm{MgCl}$ before being fixed in paraformaldehyde $4 \%$ in lysine phosphate buffer $\mathrm{pH} 7.4$ containing $2.5 \%$ of sucrose at room temperature. All subsequent steps were performed at room temperature, if not stated otherwise. Neurons were washed three times in PBS and then permeabilized for $10 \mathrm{~min}$ in PBS containing $0.5 \%$ Triton-X 100 (Sigma). Neurons were washed three times in PBS and then blocked in PBS containing 4\% goat serum (Gibco) for 30 to $60 \mathrm{~min}$. Neurons were incubated overnight at $4^{\circ} \mathrm{C}$ with guinea pig antibodies anti-MAP2 (1:3000, Synaptic Systems) in PBS containing 4\% goat serum (Gibco) to stain, respectively, the neuronal dendritic shaft for morphology and excitatory synapses for further analysis. Neurons were washed for 5 min three times in PBS and then incubated for $1 \mathrm{~h}$ with anti-guinea pig antibody coupled to AlexaFluor-488 (1:1000, Nanoprobes). Neurons were washed for 5 min three times in PBS before being postfixed in paraformaldehyde $4 \%$ in lysine phosphate buffer containing $2.5 \%$ of sucrose for $5 \mathrm{~min}$. Neurons were washed for $5 \mathrm{~min}$ three times and then stored in PBS at $4^{\circ} \mathrm{C}$ for up to three weeks until DNA-PAINT imaging.

\subsection{Spinning Disk Confocal Microscopy}

Confocal imaging was carried out with a Marianas spinning disk (3i) equipped with a $63 \times$ oil-immersion objective. A laser beam of $488 \mathrm{~nm}$ was used for excitation, in combination with a dichroic mirror (FF495-Di03-25 × 36), a clean-up filter (FF02472/30-25), and an emission filter (FF01-520/35-25). Stacks of $9.8 \mu \mathrm{m}$ with a step size of $0.7 \mu \mathrm{m}$ were acquired with an integration time of $30 \mathrm{~ms}$, a gain of 3 , and an intensification of 600 . A maximum intensity projection was generated with ImageJ. ${ }^{20}$ Adjacent regions were imaged in order to entirely cover the neuron, and the intensities were adjusted and the images stitched together in CorelDraw (CorelDrawX6).

\subsection{Sample Preparation for DNA-PAINT}

Following the sample preparation for the confocal imaging and subsequent spinning disk imaging, the samples were rinsed three times in PBS and washed two times for $5 \mathrm{~min}$ in PBS. The GluA2 subunit was indirectly labeled by a DNA-conjugated polyclonal secondary antibody (Thiol-TTATACATCTA, Eurofins Genomics; AffiniPure Donkey Anti-Mouse IgG $(\mathrm{H}+\mathrm{L}), 715$ 005-150, Jackson ImmunoResearch). Maleimide-PEG2-succinimidyl ester was used for the DNA labeling of the antibody, according to published protocols. ${ }^{12,21}$

The DNA-conjugated secondary anti-mouse antibody was diluted in blocking buffer and the cells were incubated in the solution for $45 \mathrm{~min}$ at RT. The cells were washed three times in PBS and subsequently postfixed with $2 \%$ formaldehyde (Thermo Fisher) for $5 \mathrm{~min}$ at room temperature. Fiducial markers (gold beads, diameter of $90 \mathrm{~nm}$, NANOPARTz) were diluted 1:5 in $500 \mathrm{mM} \mathrm{NaCl}, \mathrm{pH} 7.3$ and applied to the sample for $5 \mathrm{~min}$. The sample was rinsed twice in $500 \mathrm{mM} \mathrm{NaCl}$, pH7.3 before the imaging buffer was applied. For DNA-PAINT imaging, the imaging buffer contains 500 pM P1-Atto655 (CTAGATGTAT-Atto655, Eurofins Genomics) in $500 \mathrm{mM}$ $\mathrm{NaCl}$ in $\mathrm{PBS}, \mathrm{pH} 7.3 .^{14}$

\subsection{Super-Resolution Microscopy}

Immunolabeled hippocampal neurons were imaged on a commercially available N-STORM system (Nikon) composed of an Eclipse Ti-E inverted microscope (Nikon) equipped with a Perfect Focus System (Ti-PSF, Nikon) and a motorized $x-y$ stage (Nikon). Total internal reflection fluorescence (TIRF) and highly inclined and laminated optical (HILO) sheet ${ }^{22}$ configurations were adjusted using a motorized TIRF illuminator (Nikon) in combination with a $100 \times$ oil-immersion objective (CFL Apo TIRF, NA 1.49, Nikon) resulting in a final pixel size of $158 \mathrm{~nm}$. For imaging, an excitation wavelength of $647 \mathrm{~nm}$ was used, harbored in a laser combiner (MLC400B, Agilent). An optical fiber guided the laser beam to the microscope body and via a dichroic mirror (T660LPXR, Chroma) to the sample plane. Fluorescence emission were separated from excitation light via a bandpass filter (ET705/72m, Chroma) and detected by an iXon Ultra EMCCD camera (DU-897U-CS0-23 \#BV, Andor). The software NIS-Elements Ar/C (Nikon) and $\mu$ Manager $^{23}$ were used to control the setup and the camera.

\subsection{DNA-PAINT Imaging Conditions}

HILO illumination ${ }^{22}$ was used for super-resolution acquisitions with a power of 30 to $40 \mathrm{~mW}$, which was determined directly after the objective and under wide-field configuration. Videos of 50,000 frames with a bit depth of 16 bit were acquired at a frame rate of $5 \mathrm{~Hz}$ and camera read-out bandwidth of $5 \mathrm{MHz}$, a preamplification of 3 , and an electron multiplying gain of 4 .

\subsection{DNA-PAINT Simulations}

Simulations were done with Picasso using an imager strand concentration of $500 \mathrm{pM}$ and $5 \mathrm{nM}$, an integration time of $200 \mathrm{~ms}$, and a total of 50,000 frames.

\subsection{Data Analysis}

DNA-PAINT acquisitions were reconstructed with Picasso: Localize, a module of the Picasso software, ${ }^{12}$ by applying 
a minimal net gradient of 35,000. Localizations within a maximal distance of one camera pixel, showing a maximum number of transient dark frames of 15 , were linked together, resulting in a single localization event. With Picasso:Render, drift corrections were applied in essentially two subsequent fashions: first, a drift correction based on the redundant cross-correlation with a segmentation of 1000 was applied. Second, beads were manually selected, localized, and used for drift correction.

Synaptic regions were determined in ImageJ according to the signal of PSD 95. Custom written software, based on C++ and MATLAB (MathWorks), was used to filter the localization list provided by Picasso:Localize. Afterward, Picasso:Render offers the possibility of qPAINT analysis for the filtered synapses. In brief, the mean dark time for manually determined regions was determined and compared analog to the theory of qPAINT. ${ }^{12}$

\section{Disclosures}

The authors declare no competing financial interests.

\section{Acknowledgments}

We thank I. Bartnik, N. Fuerst, A. Staab, D. Vogel, and C. Thum for the preparation of cultured neurons, and N. DeußnerHelfmann for help with data processing. M. H., C. B., and E. M. S. acknowledge funding by the German Science Foundation (No. DFG CRC 902). A. S. H. is supported by an EMBO Long-term Postdoctoral Fellowship (No. ALTF 1095-2015) and the Alexander von Humboldt Foundation (No. FRA-1184902-HFST-P). E. M. S. is also funded by the Max Planck Society, an Advanced Investigator award from the European Research Council (Grant Agreement No. 743216) and DFG CRC 1080.

\section{References}

1. J. I. Arellano et al., "Ultrastructure of dendritic spines: correlation between synaptic and spine morphologies," Front. Neurosci. 1(1), 131-143 (2007).

2. W. Feng and M. Zhang, "Organization and dynamics of PDZ-domainrelated supramodules in the postsynaptic density," Nat. Rev. Neurosci. 10(2), 87-99 (2009).

3. A. J. Hughes et al., "Single-cell western blotting," Nat. Methods 11(7), 749-755 (2014)

4. S. C. Bendall et al., "Single-cell mass cytometry of differential immune and drug responses across a human hematopoietic continuum," Science 332(6030), 687-696 (2011).

5. S. Darmanis et al., "Simultaneous multiplexed measurement of RNA and proteins in single cells," Cell Rep. 14(2), 380-389 (2016).

6. Y. M. Sigal, R. Zhou, and X. Zhuang, "Visualizing and discovering cellular structures with super-resolution microscopy," Science 361(6405), 880-887 (2018)

7. M. Sauer and M. Heilemann, "Single-molecule localization microscopy in eukaryotes," Chem. Rev. 117(11), 7478-7509 (2017).

8. A. Furstenberg and M. Heilemann, "Single-molecule localization microscopy-near-molecular spatial resolution in light microscopy with photoswitchable fluorophores," Phys. Chem. Chem. Phys. 15(36), 14919-14930 (2013).

9. F. Fricke et al., "Quantitative single-molecule localization microscopy (qSMLM) of membrane proteins based on kinetic analysis of fluorophore blinking cycles," Methods Mol. Biol. 1663, 115-126 (2017).

10. G. Hummer, F. Fricke, and M. Heilemann, "Model-independent counting of molecules in single-molecule localization microscopy," Mol. Biol. Cell 27(22), 3637-3644 (2016).

11. N. Ehmann et al., "Quantitative super-resolution imaging of Bruchpilot distinguishes active zone states," Nat. Commun. 5, 4650 (2014).

12. J. Schnitzbauer et al., "Super-resolution microscopy with DNAPAINT," Nat. Protoc. 12(6), 1198-1228 (2017).
13. A. Sharonov and R. M. Hochstrasser, "Wide-field subdiffraction imaging by accumulated binding of diffusing probes," Proc. Natl. Acad. Sci. U. S. A. 103(50), 18911-18916 (2006).

14. R. Jungmann et al., "Quantitative super-resolution imaging with qPAINT," Nat. Methods 13(5), 439-442 (2016).

15. D. Nair et al., "Super-resolution imaging reveals that AMPA receptors inside synapses are dynamically organized in nanodomains regulated by PSD95," J. Neurosci. 33(32), 13204-13224 (2013).

16. U. Endesfelder et al., "A simple method to estimate the average localization precision of a single-molecule localization microscopy experiment," Histochem. Cell Biol. 141(6), 629-638 (2014).

17. C. A. Hunt, L. J. Schenker, and M. B. Kennedy, "PSD-95 is associated with the postsynaptic density and not with the presynaptic membrane at forebrain synapses," J. Neurosci. 16(4), 1380-1388 (1996).

18. G. Aakalu et al., "Dynamic visualization of local protein synthesis in hippocampal neurons," Neuron 30(2), 489-502 (2001).

19. G. Banker and K. Goslin, "Developments in neuronal cell culture," Nature 336(6195), 185-186 (1988).

20. J. Schindelin et al., "The ImageJ ecosystem: an open platform for biomedical image analysis," Mol. Reprod. Dev. 82(7-8), 518-529 (2015).

21. S. S. Agasti et al., "DNA-barcoded labeling probes for highly multiplexed exchange-PAINT imaging," Chem. Sci. 8(4), 3080-3091 (2017).

22. M. Tokunaga, N. Imamoto, and K. Sakata-Sogawa, "Highly inclined thin illumination enables clear single-molecule imaging in cells," Nat. Methods 5(2), 159-161 (2008).

23. A. Edelstein et al., "Computer control of microscopes using $\mu$ Manager," Curr. Protoc. Mol. Biol. 92, 14.20.1-14.20.17 (2010).

Carolin Böger studied physics at the University of Bielefeld, where she received her bachelor's degree in 2012 and her MSc degree in physics in 2014. Since March 2014 she has worked at the GoetheUniversity Frankfurt am Main in the group of Mike Heilemann and at the Max-Planck Institute for Brain Research in the group of Erin Schuman. Her research focuses on applying single-molecule superresolution microscopy to quantify synaptic proteins.

Anne-Sophie Hafner received her $\mathrm{PhD}$ in neuroscience from the Université de Bordeaux, France. She joined the department of Erin Schuman at the MPI for Brain Research in 2015 as a postdoctoral fellow supported by the EMBO and the Alexander von Humboldt foundations. Her research interests are synaptic function and its regulation by local protein remodeling through local protein synthesis. Her experience spans from super-resolution imaging to next-generation sequencing of subcellular compartments such as synapses.

Thomas Schlichthärle is a PhD candidate in biophysics. He did his undergraduate BSc degree in molecular medicine at the University of Tuebingen, Germany. Afterwards he moved on to pursue a MSc in molecular bioengineering at TU Dresden, Germany. During his PhD he focuses mainly on different labeling strategies for DNA-PAINT super-resolution microscopy to understand cellular processes.

Maximilian T. Strauss received his MSc degree in mechanical engineering from the Technical University of Munich and his PhD in physics from the Ludwig Maximilian University of Munich, where he applied super-resolution microscopy methods and developed imaging analysis tools. He has a strong interest in applying cutting-edge algorithms and statistical methods to bring meaningful insight into data. Currently, he is working as a postdoctoral researcher at the Max Planck Institute of Biochemistry in the group of Matthias Mann.

Sebastian Malkusch received his diploma in biophysics from the Technical University of Kaiserslautern and his PhD in chemistry from the Goethe-University in Frankfurt. He is currently a postdoctoral fellow at the Institute of Clinical Pharmacology at the Goethe-University Hospital in Frankfurt. His research interests lie in the field of quantitative molecular biology, using biophysical methods such as singlemolecule localization microscopy combined with the power of data science tools.

Ulrike Endesfelder received her diploma in physics from Bonn University in 2008 and her PhD in biophysics at the University of Bielefeld in 2012. She performed her postdoctoral trainings at Würzburg and Frankfurt University. In October 2014, Ulrike Endesfelder moved to the Max Planck Institute in Marburg, Germany, where she is 
Böger et al.: Super-resolution imaging and estimation of protein copy numbers at single synapses...

leading a research group focused on combining methods from physics and biology to understand the molecular organisation and behavior of microbial cell biology.

Ralf Jungmann received his diploma in physics form Saarland University in 2006 and his PhD from the TU Munich in 2010. Following a postdoctoral training at the Wyss Institute, he started his research group in 2014 at the Max Planck Institute of Biochemistry and Ludwig-Maximilians-Universität München. Since 2016, he has been a professor for experimental physics at the LudwigsMaximilians-University München. He combines tools from DNA nanotechnology with single-molecule fluorescence methods, targeted towards the development and application of super-resolution microscopy techniques.

Erin M. Schuman received her bachelor of arts in psychology from the University of Southern California in 1985 and her PhD in neuroscience from Princeton University in 1990. She was a postdoctoral fellow at Stanford University from 1990-1993 and then started her own lab at Caltech as an assistant professor in the Division of Biology and moved up the ranks to associate and full professor. She was also appointed as an investigator of the Howard Hughes Medical Institute from 1996-2009. In 2009, she moved to Frankfurt, Germany, to serve as a director in the Max Planck Institute for Brain Research. Her research is focused on the molecular and cellular mechanisms that allow neuronal synapses to function and exhibit plasticity in the face of the complex neuronal morphology.

Mike Heilemann received his diploma in chemistry from Heidelberg University in 2002 and his $\mathrm{PhD}$ in physics at the University of Bielefeld in 2005. Following a postdoc training at the University of Oxford (2005-2007), he started his research group in 2008, became assistant professor in Würzburg in 2011, and full professor in Frankfurt in 2012. His research interests are the development and application of super-resolution imaging and image analysis tools to understand how proteins and protein networks organize into functional units in a cell. 